\title{
A New Modular Approach for Tightly Coupled Fluid/Structure Analysis
}

\author{
Guru Guruswamy \\ NASA Advanced Supercomputing Division \\ Ames Research Center, Moffett Field, CA, U.S.A.
}

\begin{abstract}
Static aeroelastic computations are made using a $\mathrm{C}++$ executive suitable for closely coupled fluid/structure interaction studies. The fluid flow is modeled using the Euler/Navier Stokes equations and the structure is modeled using finite elements. FORTRAN based fluids and structures codes are integrated under $\mathrm{C}++$ environment. The flow and structural solvers are treated as separate object files. The data flow between fluids and structures is accomplished using $\mathrm{I} / \mathrm{O}$. Results are demonstrated for transonic flow over partially flexible surface that is important for aerospace vehicles. Use of this development to accurately predict flow induced structural failure will be demonstrated.
\end{abstract}

\section{Introduction}

Aeroelasticity that involves strong coupling of fluids and structures is an important element in the design of aerospace. vehicles. Monolithic (off-line) software to compute fluids and structures interaction studies by using low-fidelity methods such as the linear aerodynamic flow equations coupled with the modal structural equations are well advanced. 'Although these lowfidelity approaches are used for preliminary design, they are not adequate for the final design of aerospace vehicles which need a highfidelity approach to analyze complex flow/structure interactions. Modern supersonic transports that have highly swept wings can experience vortex- induced aeroelastic oscillations. ${ }^{2}$ Figure

1 illustrates flow induced structural oscillations that can occur for a typical supersonic transport when it flys in the transonic regime at moderate angles of attack. Structural oscillations can occur because of the strong coupling of unsteady leading edge vortices and wing structures. In order to predict this phenomenon, a capability to directly couple the high fidelity Euler/NavierStokes(ENS) equations with the modal structures was needed ${ }^{3}$ In Fig. 1, the strong fluid/structure coupling at 8 degrees angle of attack leads to sustained structural oscillation that degrades the flying quality of the aircraft. As another example, aeroelasticity of launch vehicles that involve strong coupling of fluids, structures and controls is an important element in the design process. ${ }^{4}$ An instability can occur soon after the launch vehicle gets separated from its carrier. The phenomenon is dominated by complex flows coupled with structural motions. From the results presented in Ref. 4 it can be concluded that low-fidelity method was not adequate to completely understand the instability phenomenon which involved non-linear flows coupled with structural motions. High fidelity equations such as the Euler/Navier-Stokes (ENS) for fluids directly coupled with finite elements (FE) for structures are needed for accurate aeroelastic computations for which these complex fluid/structure interactions exist. Using high- fidelity equations involves additional complexities results from numerics such 
as higher-order terms. Therefore, the coupling process is more elaborate when using high fidelity methods than it is for calculations using linear methods.
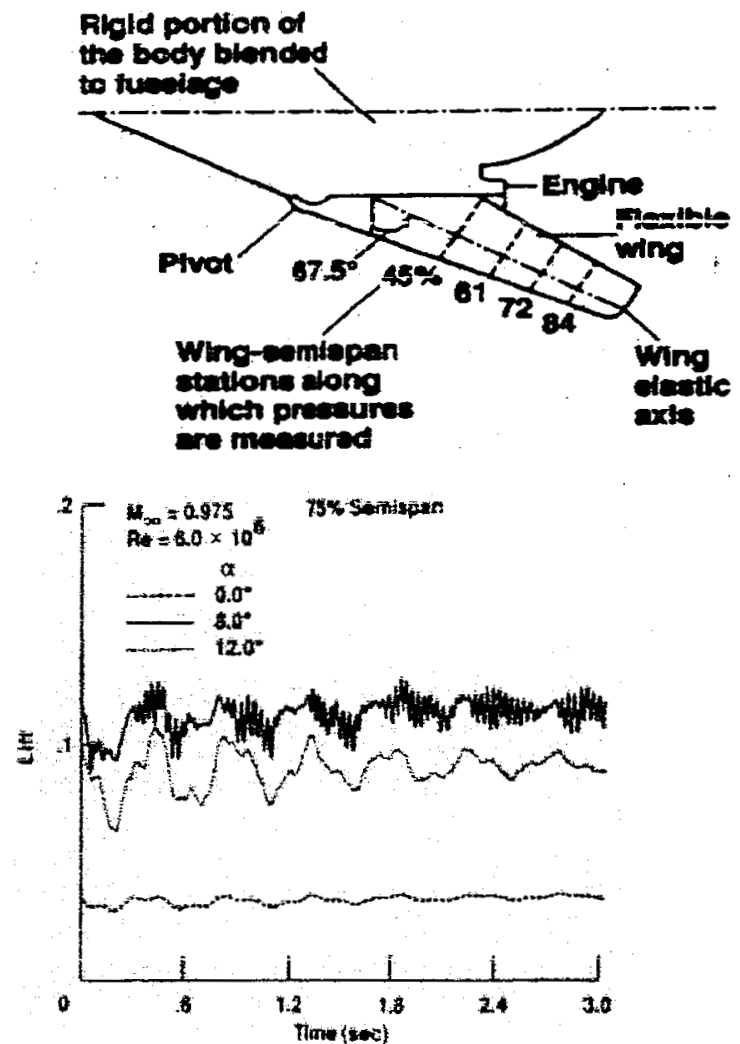

Fig. 1 Aeroelastic oscillations involving strongly coupled fluid/structures interactions

In recent years, significant advances have been made for single disciplines in both computational fluid dynamics (CFD) using finite-difference approaches 5 and computational structural dynamics (CSD) using finiteelement methods. ${ }^{6}$ For aerospace vehicles, structures are dominated by internal discontinuous members such as spars, ribs, panels, and bulkheads. The finite-element (FE) method, which is fundamentally based on discretization along physical boundaries of different structural components, has proven to be computationally efficient for solving aerospace structures problems. The external aerodynamics of aerospace vehicles is dominated by field discontinuities such as shock waves and flow separations. Finite-difference (FD) computational methods have proven to be efficient for solving such flow problems.

A major task for fluid/structure interaction studies is to combine individual discipline codes into a single computational environment. To date, monolithic serial codes such as ENSAERO $^{7}$ and $\operatorname{STAR}^{8}$, have been developed using conventional programming techniques based on FORTRAN for main frame type supercomputers. In the last decade, several monolithic software tools such as HiMAP $^{9}$, that work on single image parallel systems, were developed. These monolithic approaches are typically limited to a specific class of problems. Monolithic approaches are less accommodating to substitute of new single discipline alternative algorithms.

In the field of computer science, a new paradigm of programming technique known as problem solving environment (PSE) has evolved since the last decade. The main purpose of PSE is to provide an engineering workbench for a designer to integrate single discipline codes. Also PSE's are designed such that the application can run either on single image, parallel, or GRID (a net work of remotely distributed computers) ${ }^{\mathbf{1 0}}$ computing environments. The main feature of a good PSE is that it should require minimal changes to the application codes and provide a seamless environment. For example, SCIRun ${ }^{11}$, a 
PSE developed at University of Utah provides an environment to integrate finite element based solvers for fluids and structures.

This paper describes an approach to compute coupled static aeroelastic computations that can be ported to PSE or GRID computing environment. The approach is based on $\mathrm{C}++$ executive that controls the FORTRAN modules. A typical 3D aerospace problem of transonic flow over a thin flexible surface is analyzed.

\section{Design of $\mathrm{C}++$ Executive}

It is assumed that fluids and structure solvers are independent executables. Interfaces from fluids to structures (FTOS) and structures to fluids (STOF) are also considered as separate executables. All communications are made through $\mathrm{V} O$. This facilitates to port the present development to distributed computing. More details of this process will be given in the full paper.

During coupled calculations it is important to monitor the convergence data on the fly. Therefore a $2 \mathrm{D}$ plotting capability based on the XMGRACE ${ }^{12}$ open source software is included in the process. Also it is necessary to save data for high end graphic visualization. In this process a capability is built to save data in the FieldView ${ }^{13}$ format at user specified intervals. Fig 2 shows the flow diagram of the process.

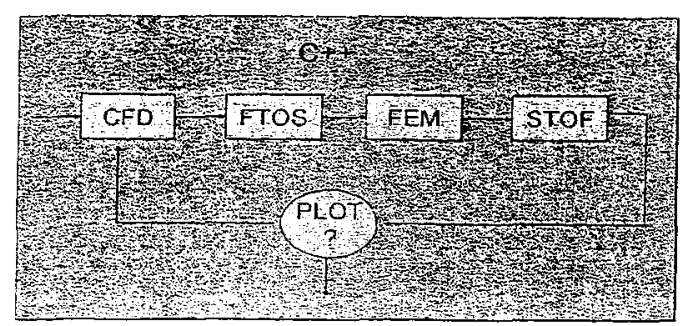

Fig. 2 Flow diagram of analysis process.

\section{Flow and Structural Equations}

In this paper computations are limited to Euler equations of motion. The strong conservative law form of the Euler equations is used for shock-capturing purposes. The equations in Cartesian coordinates in non-dimensional ${ }^{14}$ form can be written as

$$
\frac{\partial Q}{\partial t}+\frac{E}{\partial x}+\frac{\partial F}{\partial y}+\frac{\partial G}{\partial z}=0
$$

where the conserved quantity vector, $\mathrm{Q}$, and the Euler flux vectors, E, F, G, are:

$$
Q=\left[\begin{array}{c}
\rho \\
\rho u \\
\rho v \\
\rho v \\
e
\end{array}\right], E=\left[\begin{array}{c}
\rho u \\
\rho^{2}+p \\
\rho u v \\
\rho u \\
u(e+p)
\end{array}\right], F=\left[\begin{array}{c}
\rho v \\
\rho u v \\
\rho^{2}+p \\
\rho v w \\
v(e+p)
\end{array}\right], G=\left[\begin{array}{c}
\rho w \\
\rho u w \\
\rho v w \\
\rho v^{2}+p \\
w(e+p)
\end{array}\right]
$$

In Eq. (2) Cartesian velocity components $\mathrm{u}, \mathrm{v}$, and $\mathrm{w}$ are nondimensionalized by $a_{\infty}$ (the freestream speed of the sound); density $\rho$ is nondimensionlized by $\rho_{\infty}$; the total energy per unit volume, $\mathrm{e}$ is nondimensionalized by $\left(\rho \mathrm{a}^{2}\right)_{\infty}$ and the time is non-dimensionalized by $\mathrm{c} / \mathrm{a}_{\infty}$, where $c$ is the characteristic length and a is speed. Pressure can be computed from ideal gas law as

$p=(\gamma-1)\left[e-0.5 \rho\left(u^{2}+v^{2}+w^{2}\right)\right](3)$

It should be noted that " $\gamma$ "is the ratio of specific heats.

The Eq. 1 is solved to determine $\mathrm{p}$ by using streamwise upwind algorithm available in $\mathrm{GO}^{15} \mathrm{D}^{15}$ flow solver. 
The finite element approach is used to solve structural equations. In this effort a 18-degree-of-freedomplate/shell element in TRIP3D software written earlier under NASA HPCC project is used. ${ }^{16}$

The element details are shown in Fig 3. $U, V$, and $W$ are displacements degrees of freedom and Uxx, Vyy and Wzz are corresponding twist degrees of freedom in each node. The finite element equations are solved using standard procedure described in Chapter 13 (by Guruswamy) of Ref. 6.

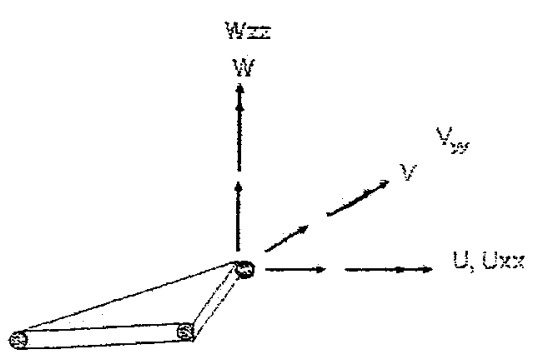

Fig. 3 18-DOF Triangular Plate/Shell Element

\section{Fluid-Structure Interfaces}

The Euler/Navier Stokes flow solvers use either patched, overset grids or unstructured grids. The surface grid data for $C F D$ is triangulated in order to interface with structures codes which is based on irregular unstructured grid. Efficient tools such as MIXSUR ${ }^{17}$ developed in association with
OVERFLOW ${ }^{5}$ are available for triangulating the complex surface grids. Figure 4 shows the possible CFD surface grids. Once triangulated data is available the next task is to interface with structures data. In this work the robust area co-ordinate approach 18 is used.

In this process, fluid grid points are identified with respect to finite element structural nodes $i, j$ and $k$ as shown in Fig 5. The force $P$ at a fluid grid point is computed using the control area associated with that point. The contribution of total force $\mathrm{P}$ at a given fluid point to the structural nodal forces $\mathrm{P}_{\mathrm{i}}, \mathrm{P}_{\mathrm{j}}$ and $\mathrm{P}_{\mathrm{k}}$ are computed using

$P_{i}=\left(P \times A_{1}\right) / A ;$

$P_{j}=\left(P \times A_{2}\right) / A ;$

$\mathrm{P}_{\mathrm{k}}=\left(\mathrm{P} \times \mathrm{A}_{j}\right) / \mathrm{A}$

where $A_{1}, A_{2}$, and $A_{3}$ are areas of the sub-triangles and $A$ is the total area in Fig 5. This procedure is repeated for all fluid grid points.

The displacements $d_{i}, d_{j}$, and $d_{k}$ from structural analysis, are interpolated to the fluid grid point using

$\mathrm{D}=\left(\mathrm{d}_{\mathrm{j}} \times \mathrm{A}_{1}+\mathrm{d}_{\mathrm{j}} \times \mathrm{A}_{2}+\mathrm{d}_{\mathrm{k}} \times \mathrm{A}_{3}\right) / \mathrm{A}$ 
TRANSFER OF CFD LOADS TO STRUCTURES

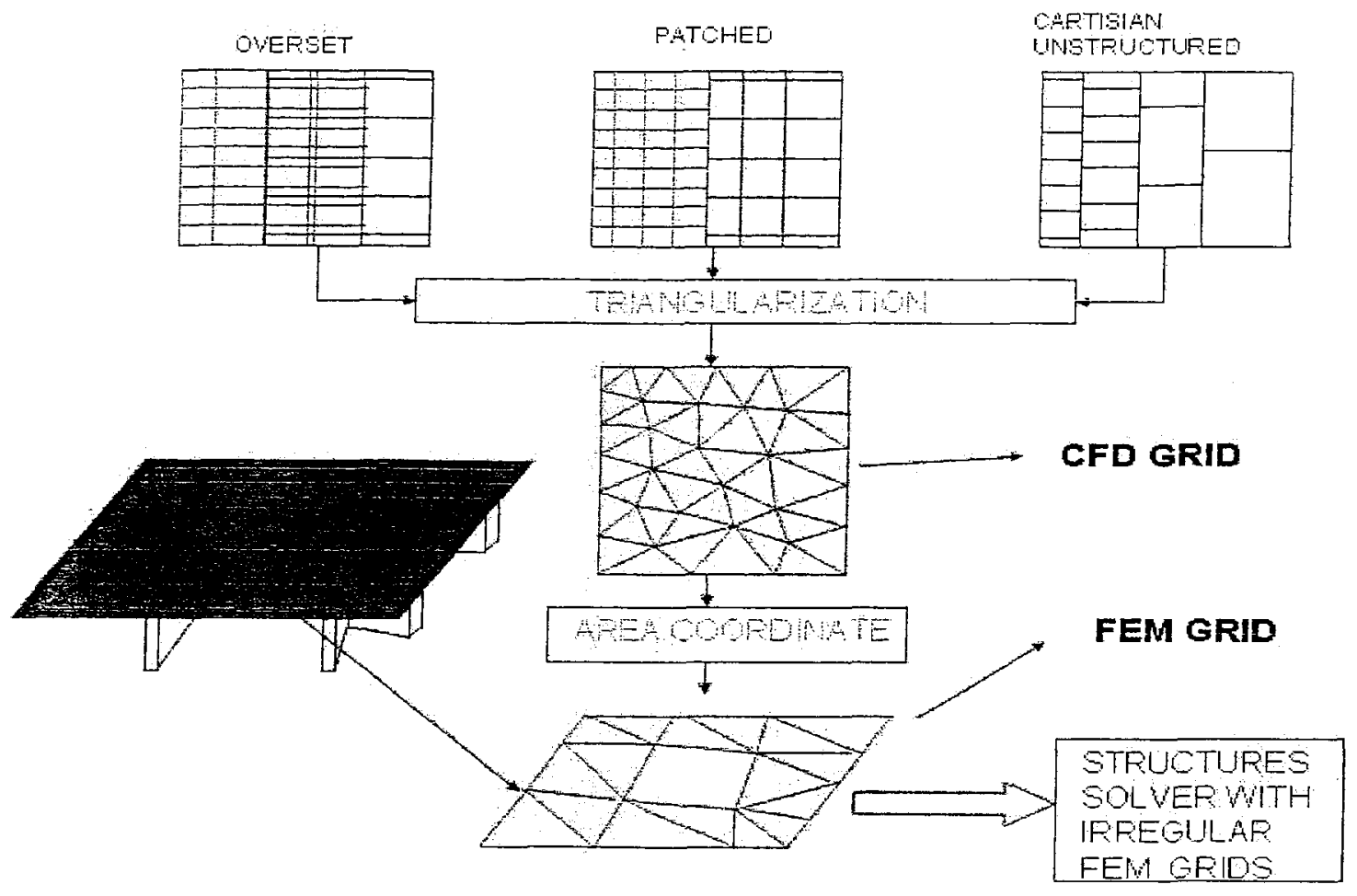

Fig. 4 Surface grid topologies of fluid and structure.

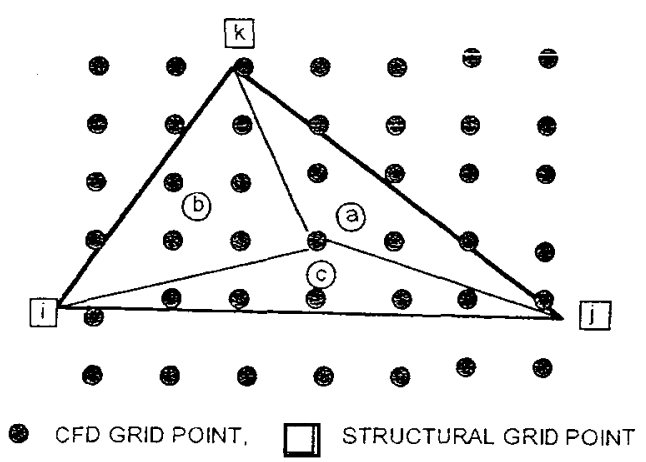

Fig. 5 Interpolation based on triangular area coordinates.

\section{Coupled Aeroelastic Computations}

Coupled aeroelastic computations play an important role in the design of aerospace vehicles. Static divergence of launch vehicles is a typical example. In order to test the process for coupled static aeroelastic computations using the
FORTRAN-based CFD and FEM codes, flow over partially flexible flat plate was selected. The flow is modeled using the streamwise - upwind 3D Euler/NavierStokes solver GO3D. ${ }^{15}$ The structures code selected was TRIP3D. ${ }^{16}$

Initially static aeroelastic computations are performed. First, loads are computed using GO3D, and then they are transferred to TRIP3D through an interface module FTOS. This interface involves interpolating loads from CFD structured surface grids to finite element nodes. As a first step, CFD structured surface grid is converted to triangular grids. Then area coordinate approach (eq. 4) is used for interfacing data between fluids and structures grids. Based on these loads, deformations and stresses are computed using TRIP3D. 
The structural deformations are interpolated back to CFD grids using area coordinate method in interface module STOF using Eq. (5). The results are monitored on the fly for convergence using XMGRACE ${ }^{12}$ plot utility.

The physical problem selected is a flow over partially flexible surface. Such configurations are common in space vehicles. Coupled fluid/structure interaction computations are often essential in designing such components. The classical example is panels of lifting surfaces that can experience flutter. ${ }^{19}$ Figure 5 shows the configuration considered for the analysis. The size of the flexible plate is assumed to 1 inch long, 0.5 inch wide and 0.005 inch thick. The size of the flow surface is 5 inch long and 1.5 inch wide. A dynamic pressure of $1.4 \mathrm{psi}$ is assumed for all computations.

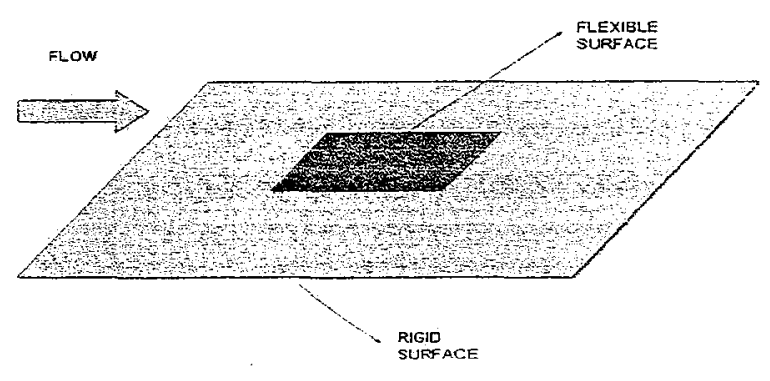

Fig. 5 Geometric details of the configuration considered.

The 3-D flow over the plate is solved using the Euler option of GO3D. A grid size of $97 \times 31 \times 34$ is used for modeling the flow surface. The portions of surface and sectional grids are shown in Fig. 6. Structural deformations and stresses are computed using 64 plate elements of TRIP3D as shown in Fig. 7. For this type of configurations both

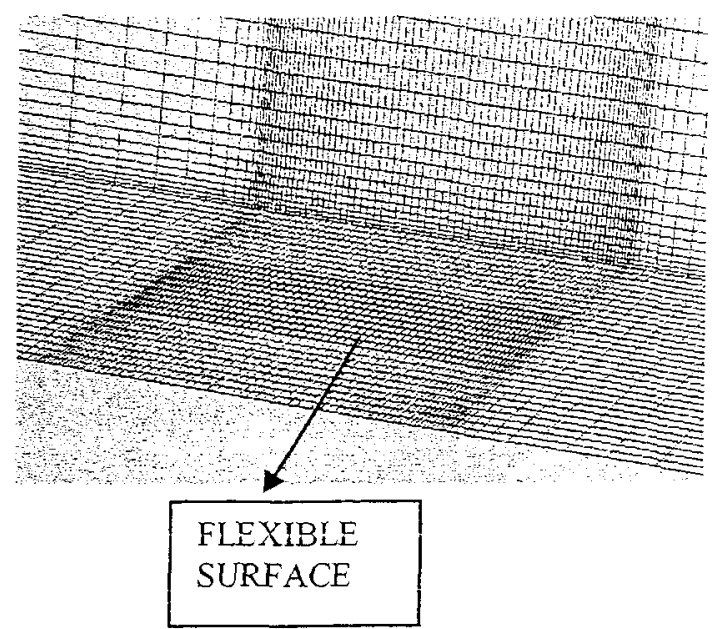

Fig. 6 Portions of CFD grid.

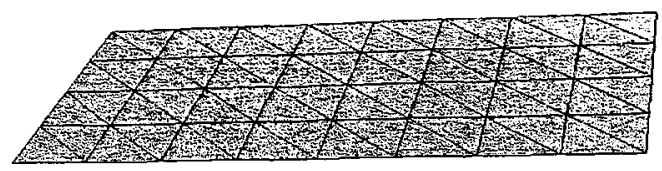

Fig. 7 FEM grid.

GO3D and TRIP3D are weil validated as independent solvers. ${ }^{16}$ For accurate computations it is important to communicate data between fluids and structures every time step particularly when one or both systems are non-linear.

Computations were made updating fluid structure information every 1,2 , and 4 time steps for a case at $\mathrm{M}=0.85$ where flow is moderately non-linear. For selected parameter structures was linear. Fig. 8 shows the effect of frequency of update on results. Updating information every 2 time step introduces significant error. Updating information every 4 steps is not acceptable.

The distribution of pressure coefficient Cp is shown in Fig 9 on half surface and vertical plane at mid span. Adaptation 


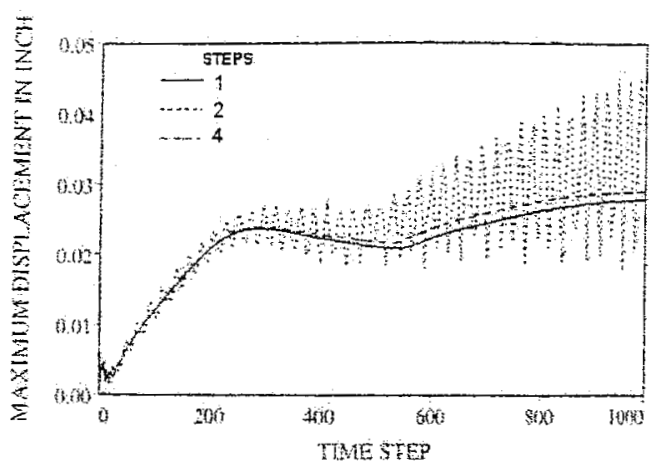

Fig. 8 Effect frequency of structural update on displacements.

of CFD grid to structural deformation can be seen in the figure. Due to flexibility of surface there is significant change in pressure distribution. The structural deformation and corresponding stress distribution can be seen in Fig 10. The variation on flow $\mathrm{Cp}$ leads to large structural stresses. The peak structural stress occurs close to $65 \%$ span near the area where $\mathrm{Cp}$ has large negative values.

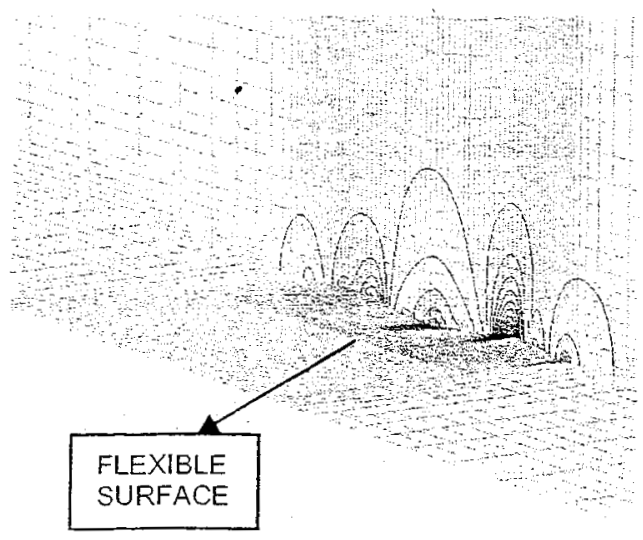

Fig. 9 Distribution of coefficient of pressure at $\mathrm{M}=0.85$.

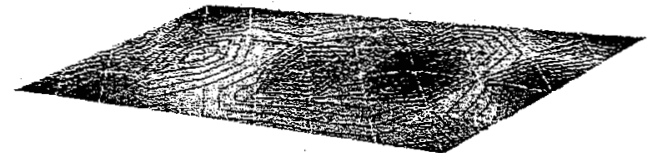

Fig. 10 Distribution of bending stresses on deformed structural surface.

Flow/structure interactions shown above can lead to unsteady flows and structural failures. The coupling will be strong during such failures. In the full paper use of present development to predict such failures will be demonstrated.

\section{Conclusions}

A procedure suitable for distributed computing to compute tightly coupled fluid structure interactions is presented. The infrastructure accommodates general CFD and FEM codes. Data management and communication is done using $\mathrm{C}++$ language which facilitates to port the present development to distributed computing system. The need to update fluid structure interface data when one of the systems is non-linear is demonstrated for a 3D problem. In the full paper use of present development to predict flow induced structural failures where the coupling is strong will be demonstrated. More details will be given in full paper.

\section{References}

1 NASTRAN User's Manual: NASA SP222 (08), June 1986.

${ }^{2}$ Dobbs, S., and Miller, G. D., "SelfInduced Oscillation Wind Tunnel Test of a Variable Sweep Wing," AIAA Paper 85-0739, Apr. 1985. 
${ }^{3}$ Guruswamy, G. P., "Vortical Flow Computations on a Blended Wing-Body Configuration," ALAA Journal, Vol 30, No 10 Oct 1992.

${ }^{4}$ Blades, E. L., Ruth, M., and Fuhrmann, H. D., "Aeroelastic Analysis of the X-34 Launch Vehicle," AIAA-99-135, Apr. 99.

${ }^{5}$ Buming, P. G., Wong, T. C., Dilley, A. D., and Pao, J. L. "Computational Fluid Dynamics Prediction of Hyper-X Stage Separation Aerodynamics, "Journal of Spacecraft and Rockets, Vol. 38, No. 6, Nov.-Dec. 2001, pp. 820-82

${ }^{6}$ Yang, T. Y., Finite Element Structural Analysis. Prentice-Hall, Inc., Englewood Cliffs, New Jersey, 1986

${ }^{7}$ Guruswamy, G. P., "ENSAERO - A

Multidisciplinary Program for

Fluid/Structural Interaction Studies," Computing Systems Engineering, Vol. 1 nos. 2-4, 1990.

${ }^{8}$ Gupta, K. K., "Development of Aeroelastic Capability," Journal of Aircraft, Vol. 33, No. 5, Sept-Oct 1999

${ }^{9}$ Guruswamy, G. P., Byun, C., M. Potsdam, M., and Farhangnia, M. “ User's Manual for HiMAP - A Multilevel Parallel Multidisciplinary Analysis Process," NASA TM-1999209578, Sept 1999.

${ }^{10}$ Foster, I., Kesselman, C., The GRID Blue Print for a new Computing Infrastructure. Morgan Kaufmann Publisher Inc, San Fracisco 2002.

${ }^{11}$ Parker, S., Weinstein, D., and Johnson, C., Modern Software Tools in Scientific Computing 1-44 Birkhuaser press, 1997
${ }^{12}$ XMGRACE, Free Software

Foundation, Inc., 675 Mass Ave, Cambridge, MA 02139, UK.

${ }^{13}$ Fieldview Version 6 Reference Manual, Intelligent Light, Lyndhurst NJ May 1999.

${ }^{14}$ Peyret, R. and Viviand, H., "Computation of Viscous Compressible Flows Based on Navier-Stokes Equations," AGRAD-AG-212, 1975.

${ }^{15}$ Obayashi, S., Guruswamy, G. P., and Goorjian P. M., "Streamwise Upwind Algorithm for Computing Unsteady Transonic Flows Past Oscillating Wings, "AIAA Jl., Vol. 29, No. 10, Oct. 1992.

${ }^{16}$ Byun, C. and Guruswamy, G. P., "Wing-Body Aeroelasticity on Parallel Computers," Il of Aircraft. $\mathrm{Vol} 33$, No 2, Mar-April 96, pp 421- 428

${ }^{17}$ Chan, W. M., and Buning, P.G., "User's Manual for FOMOCO UtilitiesForce and Moment Computation Tools for Overset Grids, "NASA TM July 1996.

${ }^{18}$ Bhardwaj, M., Kapania, R., Reichenbach and Guruswamy, G.P., "Computational Fluid Dynamics' Computational Structural Dynamics Interaction Methodology for Aircraft Wings,": AIAA $J l$ Vol 36, No 12, Dec 1998, pp 2179-2186.

${ }^{19}$ Korenecki, A., Dowell, E. H., and O'Brien, J., "On the aeroelastic Instability of Two-Dimensional Panels in Uniform Incompressible Flow," $J l$ of Sound and Vibration, Vol 47 (1976) pp. 163-178. 